\title{
4 Nameninventar
}

Zusammenfassung: Im Kapitel wird das erstellte Inventar mit über 520 SNToken, die zwischen 1884 bis 1945 in nachweislich kolonialer Motivik in den städtischen Raum von Groß- und Mittelstädten der deutschen Metropole verfügt wurden, beschrieben. Koloniale Namenvergabepraktiken können als ortsübergreifend-nationale Prozesse beschrieben werden, die sich in nahezu 100 Großund Mittelstädten sowie einzelnen Ortspunkten der am Ende der 1930er Jahre annektierten Gebiete nachweisen lassen. Dabei kann gezeigt werden, dass der maßgebliche Anteil der linksköpfigen Erstglieder als relevant und unmittelbar auf die Kolonien bezogen verstanden wurde.

Die notwendigen Arbeitsschritte, wie kolonial motivierte Straßenbenennungen von 1884 bis 1945 identifiziert werden können, wurden exemplarisch anhand zweier Städte des zusammengestellten Ortskorpus gezeigt. Sie verdeutlichen, dass die Identifizierungsaufgabe der Kolonialismen bis 1945 ein zeitintensives Unterfangen darstellt, das nur durch die additive Informationsgewinnung aus unterschiedlichsten historischen Quellen und Quellengattungen erfolgreich durchgeführt werden kann. Das erstellte Ortskorpus an über 400 bzw. 370 (unter Berücksichtigung der Eingemeindungen) Groß- und Mittelstädten inklusive der am Ende der 1930er Jahre annektierten Städte mit über 20.000 Bewohnerinnen und Bewohnern wurden bis 1945 hinsichtlich etwaiger kolonialer Benennungen in Straßennamenlexika, historischen Adressbüchern sowie Stadtplänen u. dgl. überprüft. Dabei konnte ein Inventar mit einem beachtlichen Umfang an über 520 (527) SN-Token zusammengestellt werden: Trotz der nur drei Jahrzehnte andauernden faktischen Kolonialepoche in der Zeit des Kaiserreichs handelt es sich bei kolonialen Namenvergabepraktiken um usuelle sprachhistorische Phänomene, die ortsübergreifend im (Alltags-)Raum des Deutschen Reichs Verwendung fanden. Das erhobene Inventar wird im Appendix 1 tabellarisch unter Angabe der Zuweisung der jeweiligen tokenbezogenen Sprachdaten zu einem (städtischen) Raum dargelegt.

Die linksköpfigen Erstglieder der erhobenen Konstruktionen waren dabei ausschlaggebend für die Identifizierung kolonial motivierter SN-Token aus der Perspektive ex ante, die nicht nur ausdrucksseitig, sondern auch hinsichtlich der inhaltsseitigen Zuschreibung zu Kolonialismus und kolonialen Themen quellenbasiert untersucht wurden. Die Koloniallexika (vgl. Kausch 1903, Schnee 1920) konnten als erstes (von notwendigen weiteren quellenbezogenen) Prüfverfahren genutzt werden, um herauszufinden, inwieweit etwaige sekundäre Namen bzw. deren Erstglieder relevant und unmittelbar auf die Kolonialzeit 
bezogen verstanden wurden. Umgekehrt bleibt für das erstellte Inventar der über 520 SN-Token festzuhalten, dass nahezu alle Erstglieder entweder als eigene Lemmata in den Koloniallexika vorzufinden sind oder im Beschreibungsteil anderer Lemmata erwähnt werden. Eine Ausnahme stellen alle diejenigen 30 Konstruktionsmuster dar, die auf Paul von Lettow-Vorbeck referieren. Das kolonialpolitische Wirken Lettow-Vorbecks fällt maßgeblich in die Endzeit der de facto-Kolonialzeit: „Im Okt. 1913 erfolgte seine Ernennung (als Oberstleutnant) zum Kommandeur der Schutztruppe in Kamerun, im April 1914 zum Kommandeur der Schutztruppe in Deutsch-Ostafrika“ (Gründer 1985: 358-359). Dass zur Person Paul von Lettow-Vorbeck weder eigene Lemmata noch Informationen innerhalb von Beschreibungsteilen anderer Einträge vorliegen, ist damit zu begründen, dass die Koloniallexika bereits vor Kriegsbeginn abgeschlossen wurden. Auch bei den unter zehn verbleibenden singulären Konstruktionen, deren Erstglieder nicht als eigene Lemmata oder in Beschreibungsteilen anderer Lemmata auftauchen, konnte die inhaltliche Zuschreibung $\mathrm{zu}$ Kolonialismus und kolonialen Themen quellenbasiert nachgewiesen werden: Das gilt auf der Token-Ebene bspw. für die Benennung Nettelbeckstraße in München-Trudering, die sich in dem einzelortsbezogenen Fall auf sein koloniales Engagement beziehen sollte (Kap. 3.5). Auch die Erstglieder der in Braunschweig verfügten singulären Konstruktionen Albert-Voigts-Weg, HermannBlumenau-Straße und Otto-Finsch-Straße finden keine Erwähnung in den Koloniallexika. Nichtsdestotrotz wird den jeweiligen Personen zeitgenössisch eine lokalpatriotische Relevanz in Bezug auf Kolonialismus zugeschrieben; so findet sich im zeitgenössischen Adressbuch der Hinweis, dass sich die drei SN-Token in kommemorativer Intention auf „Braunschweiger [...] Kolonialpionier[e]“ (Adressbuch Braunschweig 1940 III: 39, 148, 239) beziehen sollten. Nicht zuletzt - das wurde bereits in Kap. 3.3.5 beschrieben - legen Stadtpläne themenkohärente Clusterbenennungen offen, sodass auch solche Verfügungen ins Namenkorpus aufgenommen werden konnten, deren Erstglieder nicht in den Koloniallexika auftauchen, sich aber dennoch nachweislich auf unmittelbare kolonialzeitbezogene Wissensordnungen beziehen. Das betrifft bspw. das SNToken Transvaalstraße in Oranienburg, das zeitgleich zusammen mit weiteren gebündelten Namenmuster, für die eindeutige koloniale Kommemorationen festzustellen sind, für neu angelegte Straßenzüge verfügt wurde (Abb. 9).

Der Stadtplan veranschaulicht das in unmittelbarer Umgebung des städtischen Krankenhauses verfügte Kolonialcluster: Bis auf das SN-Token Transvaalstraße sind die Erstglieder aller weiteren Benennungen (Dualastraße, Kamerunstraße, Lüderitzstraße, Otavistraße, Swakopmunder Straße, Taborastraße, Togostraße, Windhukstraße) als eigene Lemmata in den Koloniallexika vertreten; die Namen referieren auf einen Kolonialakteur und auf Orte der kolonisierten Räume in Afrika. 


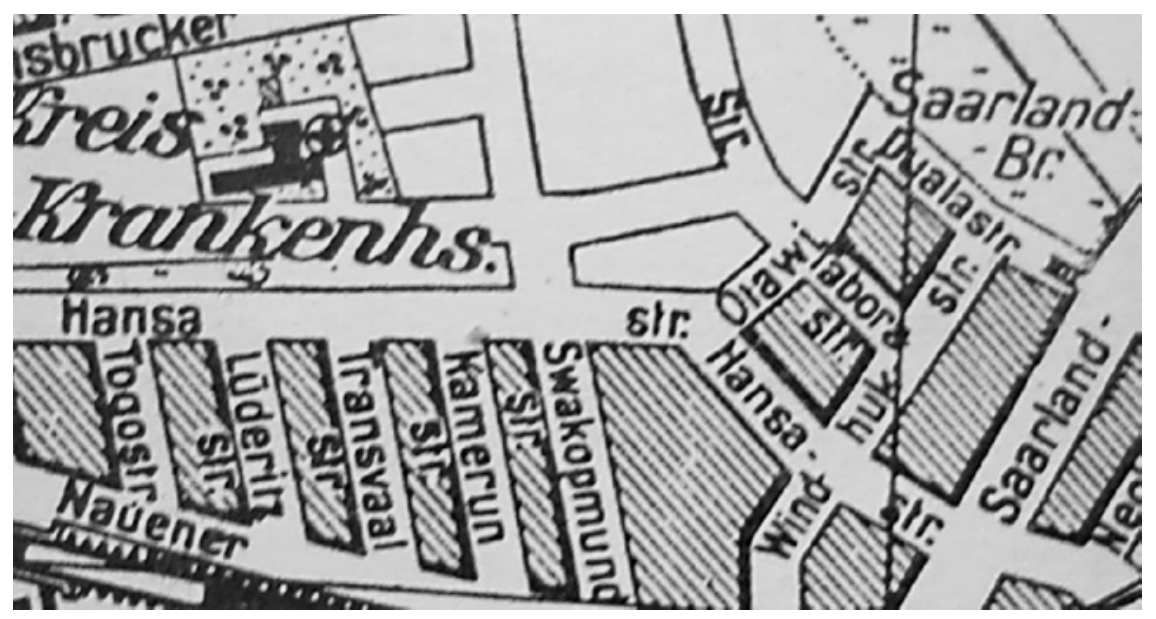

Abb. 9: Stadtplan Oranienburg (1940), Ausschnitt (heutiges) Ärzteviertel.

Alle neun Benennungen sind erstmalig am Ende der 1930er im Straßenverzeichnis (vgl. Adressbuch Oranienburg 1937/38: 117-191) aufgeführt, allerdings ohne Benennungsmotiviken. Auch die Namenverfügung Transvaalstraße ist ins Nameninventar dieser Arbeit aufzunehmen: Bei den Straßenzügen der SN-Token Lüderitzstraße, Kamerunstraße, Swakopmunder Straße, Togostraße, Transvaalstraße und Windhukstraße handelt es sich um Parallelstraßen, die alle von Süden auf den Straßenzug des schon seit den 1920er Jahre belegten SN-Tokens Hansastraße (vorher: Berliner Feld) mündeten. ${ }^{93}$ Die Straßen der Benennungen Otavistraße, Taborastraße und Dualastraße wurden in unmittelbarer Nähe nördlich davon angelegt. Bei allen SN-Token handelt es sich um zeitgleich erfolgte Verfügungen aus der Zeit des Nationalsozialismus, die 1936 für neu erschlossene Straßenzüge erfolgten. Wenngleich die Provinz Transvaal nie in deutschem Kolonialbesitz war, kam es zwischen 1895 und 1897 zwischen der Kapkolonie unter britischer Herrschaft und der benachbarten Südafrikanischen Republik, auch Transvaal genannt, zu Auseinandersetzungen, bei denen auch das Deutsche Kaiserreich nicht unbeteiligt blieb (vgl. Clark 2009: 131-132). Dass Transvaal, unter anderem durch die hohe Zahl deutscher Siedler vor Ort, durchwegs ein Ort kolonialer Imaginationen des Deutschen Reichs gewesen ist und innerhalb des Kolonialdiskurses eine nicht unerhebliche Rolle gespielt hat, wird auch in zeitgenös-

93 Heutiger Name: Robert-Koch-Straße. Herzlicher Dank geht an C. Becker vom Stadtarchiv Oranienburg. 
sischen Quellen zum Ausdruck gebracht. Für Transvaal sind in der DSDK 174 Treffer zu verzeichnen. Auch die nationalliberale Zeitschrift Die Grenzboten setzt sich mehrmals mit der Provinz auseinander.

Die Lage der Dinge in Transvaal. [Fettdruck im Original] Die Wichtigkeit der TransvaalFrage für Deutschland wird derjenige verstehen, der die Wichtigkeit überseeischer Kolonisation und insbesondere die Bedeutung von Südafrika für letztere versteht; [...] Überall wo wir noch in den letzten Jahrzehnten uns hätten niederlassen können, ist uns England zuvorgekommen und hat das von Anderen bisher verschmähte Küsten- oder Inselland besetzt, damit wir es nicht besetzen können; (Die Grenzboten 1880: 485)

Deutschlands Beziehungen zu Transvaal. [Fettdruck im Original] [...] Es hat sich also in Transvaal ein weites Gebiet für den deutschen Unternehmensgeist erschlossen. [...] Vor allen Dingen unterscheiden sich aber die Deutschen vorteilhaft dadurch von den englischen Zugvögeln, daß sie in überwiegender Zahl im Lande seßhaft geworden sind, sei es als Arbeiter, Handwerker, Ackerbauer oder Kaufleute. [...] Wie warm und anerkennend waren die Worte, die Präsident Krüger am 27. Januar 1895 in seinem Trinkspruch auf unsern Kaiser den Deutschen spendete! [...] ich hoffe, daß sich Transvaal immer fester an Deutschland anschließen wird. (Die Grenzboten 1896: 305, 308-309)

Ziel der vorliegenden Studie besteht gerade nicht darin, die Mikrotoponyme im Rahmen traditioneller Kolonialismusforschung hinsichtlich historischer Faktizität für die allgemeine Kolonialgeschichtsschreibung auszuwerten. Insofern sind derartige Einzelphänomene zur Ermittlung der sprachlich-diskursiven Konstruktion kolonisatorischer Selbstzuschreibungskonzepte von Relevanz und in die Analysen mit einzubeziehen.

Das erstellte Nameninventar an über 520 SN-Token weist einen bemerkenswerten Umfang auf, die sich wiederum auf eine beachtliche Zahl an Groß- und Mittelstädten des erstellten Ortskorpus verteilen. Bei der Vergabe kolonialer Straßennamen im Deutschen Reich handelt es sich um ortsübergreifendnationale sprachliche Prozesse, die über die administrativen und/oder handelsspezifischen Kolonialmetropolen in der Zeit des Kaiserreichs wie Berlin und Hamburg (Fn. 10, Kap. 2.1.4) oder Bremen, die in nationalsozialistischer Zeit propagandistisch inszeniert wurde (Fn. 54, Kap. 2.3), eine ganze Reihe weiterer Städte miteinschließt.

Es ist darauf hinzuweisen, dass im Rahmen der Erstellung eines solchen, vorrangig auf historische Quellen und Quellengattungen angewiesenen Nameninventars zwangsläufig einzelne Wissenslücken bestehen bleiben: Für singuläre Städte des Ortskorpus gestaltete es sich innerhalb des betreffenden Zeitraumes als unmöglich, entsprechende zeitgenössische Benennungsakten, Adressbücher, Stadtpläne $\mathrm{u}$. dgl. zu sichten. Diese Wissenslücken betreffen nicht alle Gebiete östlich der Oder-Neiße-Grenze, sondern vor allem diejenigen Städte, die innerhalb 
der am Ende der 1930er Jahre errichteten Reichsgaue zu verorten sind. Insbesondere für Städte der im Zuge des Polenfeldzugs besetzten Gebiete liegen nur für einen Bruchteil der Untersuchungsorte Adressbücher, Stadtpläne u. dgl. vor. ${ }^{94}$ Für Städte, die 1938 bzw. 1939 durch die Besetzung des Sudetenlandes bzw. der Errichtung des Protektorats Böhmen und Mähren dem Deutschen Reich angegliedert wurden, gestaltete sich das Identifizierungsverfahren dagegen als weniger problematisch: So hat bspw. die Überprüfung eines von der „Kurverwaltung Karlsbad“ herausgegebenen Stadtplans von 1940 ergeben, dass noch im gleichen Jahr der im Zuge des Münchner Abkommens besetzten Stadt (vgl. Kalousková angenommen) ein SN-Token verfügt wurde, das sich auf den Reichskommissar und Gouverneur von Deutsch-Ostafrika bezog (Abb. 10).

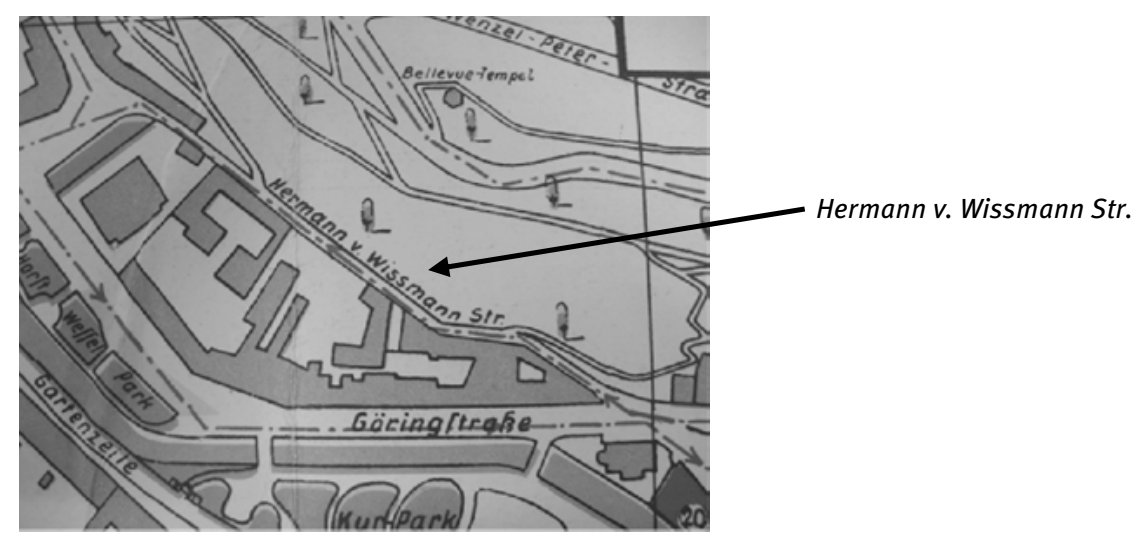

Abb. 10: Plan Karlsbad [Karlovy Vary] (1940).

Für Asch [Aš] ist zu Beginn der 1940er Jahre die Veröffentlichung eines Einwohnerbuchs zu verzeichnen, in dem das SN-Token Lüderitzstraße ohne Angabe einer Benennungsmotivik erstmalig aufgeführt wurde (vgl. Adressbuch Asch 1941: 80). Kalousková (angenommen) zeigt unter Einbezug weiterer zeitgenössischer Akten auf, dass es sich dabei um ein kolonialzeitbezogenes SN-Token handelt, das auf den Kolonialakteur Adolf Lüderitz referieren sollte. Sie wurde daher ins Inventar der vorliegenden Arbeit aufgenommen. Für eine ganze Reihe an Städten des als „Reichsprotektorat Böhmen und Mähren“ besetzten Gebietes liegen historische

94 So sind bspw. keine historischen Adressbücher und/oder Stadtpläne für die Städte Bendzin [Będzin], Chrzanow bzw. Krenau [Chrzanów], Laurahütte [Siemianowice Śląskie], Jaworzno [Jaworzno], Teschen [Český Těšín], Zgierz bzw. Görnau [Zgierz], Zdunska Wola [Zduńska Wola] und Zawierce bzw. Warthenau [Zawiercie] innerhalb der relevanten Zeitspanne verfügbar. 
Stadtpläne von Seiten des in Prag eingerichteten „Landesvermessungsamtes Böhmen und Mähren“ vor. ${ }^{95}$ Die Pläne zeigen nur für die jeweiligen Zentren der Altstadt geschwärzte deutschsprachige Straßennamen an. Man gewinnt den Eindruck, dass diese Überschreibungsprodukte zuvor getilgter tschechischsprachiger Straßennamen darstellen. Die Peripheriebereiche werden in den Karten ausgespart. Es ist davon auszugehen, dass die tschechischsprachigen Straßennamen außerhalb der altstädtischen Zentren nicht von derartigen Tilgungsmaßnahmen betroffen gewesen sind. Abbildung 11 zeigt einen Ausschnitt des Zentrums der Stadt Prerau [Přerov] am Anfang der 1940er Jahre:

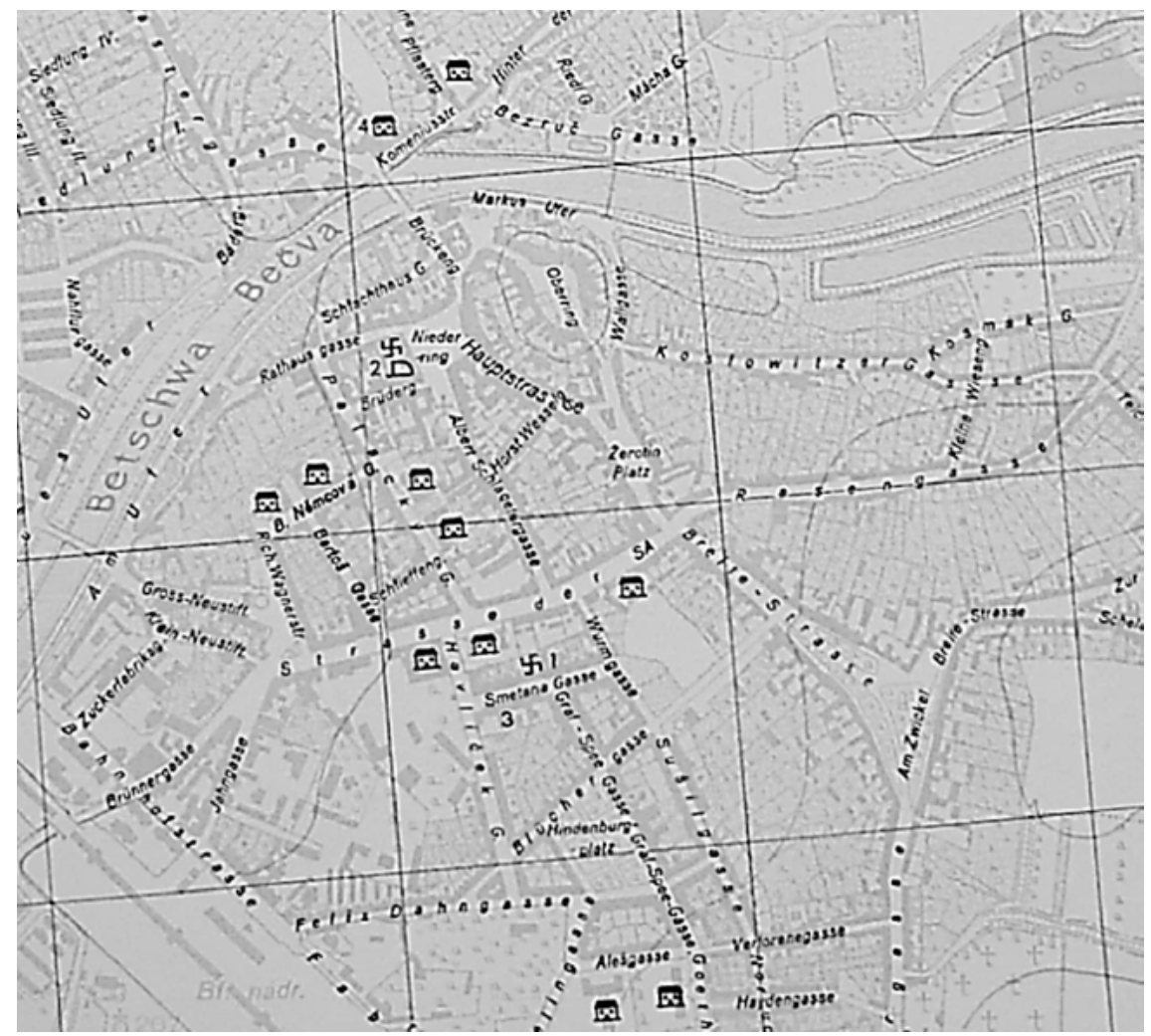

Abb. 11: Plan Prerau [Přerov] (1943).

95 Man vgl. dazu die entsprechenden Stadtpläne für (Böhmisch-)Budweis (1943), Iglau (1943), Jungbunzlau (1943), Kladno (1943), Kolin (1943), Prerau (1943) und Proßnitz (1943), die in der Kartenabteilung der SBBPK systematisch hinsichtlich etwaiger kolonial motivierter SN-Token geprüft wurden. 
Betrachtet man die geschwärzten deutschsprachigen Umbenennungsprodukte, dann sind orientierende Straßennamen $\mathrm{zu}$ verzeichnen, die als georeferierend beschrieben werden können (Am Ufer, Breite-Strasse, Hauptstraße, Rasengasse u. dgl.) oder sich auf Infrastruktureinrichtungen (Bahnhofstraße, Rathausgasse, Zuckersfabriksgasse $\mathrm{u}$. dgl.) beziehen. Daneben ist auch eine ganze Reihe an Straßennamen mit offensichtlich kommemorativ intendierten Funktionen (etwa Blüchergasse, Graf-Spee-Gasse, Hindenburgplatz, Horst Wessel Gasse, Schlieffengasse) festzustellen, bei denen es sich offenbar nicht um reine Übersetzungen handelte: Sie beziehen sich vorrangig auf Personen, die innerhalb des preußischen Militarismus einzuordnen sind. Die Sichtung weiterer Stadtpläne von 1943 für die jeweiligen betreffenden Ortspunkte zeigen eine ganz ähnliche Verteilung deutschsprachiger Straßennamen mit Orientierungsfunktion und kommemorativ intendierten Funktionen auf.

In Bezug auf etwaige kolonial motivierte Straßennamenverfügungen für Städte des errichteten Protektorats konnte in den jeweiligen verfügbaren Stadtplänen unter anderem das SN-Token Lüderitzgasse für Iglau [Jihlava] ausfindig gemacht werden:

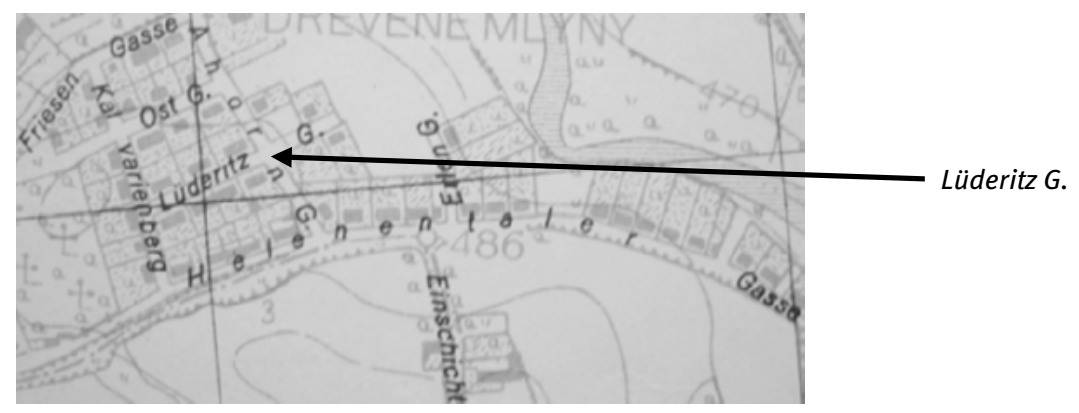

Abb. 12: Plan Iglau [Jihlava] (1943).

Kalousková (angenommen) konnte nachweisen, dass es sich bei dem SN-Token der zweitgrößten deutschsprachigen Enklave im Protektorat Böhmen und Mähren ebenfalls um eine kolonial motivierte Benennung handelt, die sich bezeichnungsmotivisch auf Adolf Lüderitz und dessen koloniale Inbesitznahme Südwestafrikas (vgl. Schnee 1920 II: 465) beziehen sollte. Auch im historischen Kern von Prag, das von nationalsozialistischer Seite zur Hauptstadt des Protektorats ausgerufen wurde, wurde die Verfügung des SN-Tokens Lüderitzufer geplant, letztendlich aber nicht umgesetzt (vgl. Kalousková 2014: 34). Aus diesem Grund wurde das SN-Token nicht ins Inventar der vorliegenden Studie aufgenommen. 
Dass letztendlich nur für etwa 15 Städte des über 370 Groß- und Mittelstädte beinhaltenden Ortskorpus (unter Berücksichtigung der Eingemeindungen) keinerlei historische Quellengattungen gesichtet werden konnten, ist als ein Erfolg zu verzeichnen. ${ }^{96}$ Diese große Abdeckung liegt mitunter auch an der großen Unterstützung der Inventarisierungsarbeiten durch die Mitarbeiterinnen und Mitarbeiter unzähliger ortsansässiger Stadtarchive. Sie unterstützten die Zusammenstellung des Nameninventars durch hilfreiche Auskünfte aus ihren jeweiligen Archivbeständen, indem sie für den Untersuchungszeitraum historische Benennungsakten, Adressbücher und Stadtpläne in Bezug auf etwaige koloniale Straßennamen sichteten und diese zum Teil sogar als Scan für die vorliegende Arbeit zur Verfügung stellten. Ihnen gilt ein besonderer Dank.

Obwohl Kleinstädte nicht ins zu untersuchende Ortskorpus mit aufgenommen wurden, ergaben stichpunktartige Recherchen, dass selbst in Orten unter 20.000 Einwohnern kolonial motivierte Straßenbenennungen bis $1945 \mathrm{zu}$ konstatieren sind. So ist bspw. für die Kleinstadt Bad Oeynhausen am Ende der faktischen Kolonialzeit die Benennung Lettow-Vorbeck-Straße zu verzeichnen (vgl. Pöppinghege 2007: 52-53). Weidner zeigt für eine ganze Reihe an Kleinstädten in Westfalen und Lippe Benennungen in nationalsozialistischer Zeit auf, die sich ebenfalls in kommemorativer Intention auf Paul von LettowVorbeck beziehen sollten. ${ }^{97}$ Für die ebenso im heutigen Gebiet Westfalen-Lippe zu verortende Kleinstadt Plettenberg wurde Ende der 1930er Jahre ein Kolonialcluster mit einer Vielzahl kolonial motivierter SN-Token (Askaristraße, KarlPeters-Straße, Kamerunstraße, Ostafrikastraße u. dgl.) für projektierte Straßen beschlossen, die allerdings nicht umgesetzt wurden. ${ }^{98}$ Ortsübergreifende Aussagen über koloniale Namenvergabepraktiken in Kleinstädten können in der vorliegenden Arbeit nicht getroffen werden. Das gilt ebenso für die bis 1945 selbstständigen Gemeinden: So wurden bspw. die rezenten, in den 1930er Jahren nach kolonialzeitlichen Akteuren erfolgten Benennungen Gustav-NachtigalStraße und Lettow-Vorbeck-Straße in Erfenbach nicht ins Nameninventar aufgenommen, da die Eingemeindung Erfenbachs in die kreisfreie Stadt Kaiserslautern erst 1969 im Zuge der rheinland-pfälzischen Verwaltungsreform erfolgte.

96 Dazu zählen bspw. neben den bereits in Fn. 94 dargelegten Orte auch die Städte Herten und die Lutherstadt Eisleben: Für diese Ortspunkte stehen keine Straßennamenlexika, Adressbücher, Stadtpläne u. dgl. zur Verfügung. Auch die Versuche einer Kontaktaufnahme mit den jeweiligen Stadtarchiven blieben ohne Erfolg.

97 Bspw. für Bünde, Halle in Westfalen, Plettenberg und Rheine (https://www.lwl.org/westfaelische-geschichte/nstopo/strnam/Begriff_192.html, Abruf am 28/06/2019).

98 https://www.lwl.org/westfaelische-geschichte/nstopo/strnam/Kommune_172_Existierend. html, Abruf am 28/06/2019. 
Auch die für Vororte festzustellenden Kolonialismen bleiben unberücksichtigt. Ein solches Beispiel stellen die bis 1905 in Karlshorst eingeschriebenen SNToken Bothaallee, Dewetallee, Ohm-Krüger-Straße, Warmbader Straße und Waterbergstraße dar (vgl. Ahlfänger 2009: 4):

Sämtliche Straßen im „Burenviertel“ erinnerten an Orte, Gegenden und Personen, die mit dem kolonialen Südwesten Afrikas (Namibia und Südafrika) verbunden waren. Ausschlaggebend dafür war die Begeisterung für die Buren, die im Zweiten Burenkrieg (1899 bis 1902) nach äußerst grausamen Kämpfen den Briten unterlagen. (Ahlfänger 2010: 4)

Karlshorst wird seit seiner späten Gründung 1895 bis zur Ausgabe 1921 im Berliner Adressbuch als Vorort von Berlin und Bestandteil der Gemeinde Friedrichsfelde geführt. Im dazugehörigen Straßenverzeichnis (vgl. Adressbuch Berlin 1921 V) werden darüber hinaus die weiteren SN-Token Aviser Str., Bloomfontainer Straße, Burenring, Kalaharistraße, Keetmanshooper Straße u. dgl. für unbebaute Straßenzüge angegeben, „doch die Straßen wurden nicht bebaut oder gar nicht erst angelegt, so dass sie Mitte der 30er Jahre aus den Verzeichnissen verschwanden“ (Ahlfänger 2010: 4). Jene Benennungen wurden nicht zentral von Berlin aus geplant. Stattdessen waren für die bis 1920 festzustellenden Straßenplanungen private Erschließungs- und Baugesellschaften verantwortlich. ${ }^{99}$ Das gilt bspw. auch für die noch vor 1900 verfügte Benennung Wissmannstraße in Berlin-Rixdorf bzw. (seit 1912) Neukölln (vgl. Adressbuch Berlin 1910 III: 936). Die Kolonialismen in Berlin-Dahlem wurden dagegen berücksichtigt und ins Nameninventar aufgenommen: Wenngleich Dahlem ebenso bis 1920 ein Berliner Vorort darstellte (vgl. Adressbuch Berlin 1921 V) und erst dann nach Groß-Berlin eingemeindet wurde, stellte Dahlem seit Anfang des 20. Jahrhunderts eine „Kgl. [d. i. königliche] Domäne“ (Adressbuch Berlin 1908 V: 164) dar. Seit 1901 wurde eine besondere Kommission mit Sitz in Berlin gebildet, die für den Vorort und dessen infrastruktureller Erschließung zuständig war. Seit Beginn des 20. Jahrhunderts erfolgte die Straßenbenennung demnach bereits von staatlicher Seite aus (vgl. Engel 1984: 29-31). Die in nachweislich kolonialer Motivik erhobenen SN-Token Lansstraße, Iltisstraße und Takustraße wurden noch in der faktischen Kolonialzeit von der „Kommission zur Aufteilung der Domäne Dahlem“ verfügt. ${ }^{100}$

Das für nahezu 100 Groß- und Mittelstädte sowie einzelne Orte der am Ende der 1930er Jahre annektierten Gebiete erhobene Inventar ermöglicht nicht nur erstmalig ortsübergreifend-inventarbezogene Analysen, sondern schafft auch

99 Herzlicher Dank geht an J. Ahlfänger für die umfangreichen Informationen.

100 https://berlin.kauperts.de/Strassen/Iltisstrasse-14195-Berlin, Abruf am 28/06/2019. 
die Voraussetzung für darauffolgende Generalisierungen über die Grenzen städtebezogener Teilinventare. Die Namen sind als serielle Prozesse im Hinblick auf inventarbezogene Musterhaftigkeiten zu untersuchen, denn „nur eine systematische Erfassung [...] des möglichst umfänglich erfassten kolonialen Toponomastikons kann die symbolische Kolonialterritorialisierung überhaupt erst aufzeigen und damit verständlich machen" (Stolz und Warnke 2018b: 55). Die Beantwortung der innerhalb bisheriger kolonialtoponomastischer Untersuchungen formulierten übergeordneten Frage nach „diskursive[n], das heißt über das Einzeltoponym hinausreichende[n] Wissensordnungen“" (Stolz und Warnke 2018b: 47) wird im Folgenden für das koloniale Namenprojekt der Metropole ausgeweitet. Die Beantwortung der zu Beginn der Arbeit vorgelegten Fragestellungen erfordert eine inventarbezogene strukturierte Annotation nach sprachwissenschaftlichen Kriterien, um über die einzelnen Ortspunkte hinausgehende inventarbezogene Untersuchungen zu ermöglichen. 